\title{
Relay based Clustering Protocol for WSN
}

\author{
Jayanthi K Murthy \\ BMS College of Engineering, Bangalore
}

\author{
Sambasiva Rao V \\ PES Institute of Technology, Bangalore
}

\begin{abstract}
Energy minimization and increase in lifetime have become the key factors for data transmission in a wireless sensor network. An energy efficient protocol called Energy Efficient Relay Based Multi-hop Clustering (EEMRC) that balances the energy consumption in clusters is implemented with the help of relay nodes. Comparison of the proposed protocol with the standard LEACH protocol yielded results which showed a large improvement in network lifetime.
\end{abstract}

\section{General Terms}

Wireless Sensor Networks, Wireless Communication

\section{Keywords}

Energy efficient, multi hop network, network lifetime, relay based.

\section{INTRODUCTION}

The advances in wireless communication embedded computing and electronics have enabled the development of the low-power wireless sensor network (WSN) technologies. A sensor network is a network of tiny, lightweight, batteryoperated devices, known as sensor nodes. Each sensor node in a sensor network is also equipped with wirelesscommunication devices. The replacement of heavy weighted wired technology with many small, low cost wireless sensor nodes ensure a wide range of applications for WSNs. Each sensor node in a sensor network can sense only its vicinity. The readings from all sensor nodes can be gathered, possibly at a distant location, to get a broader picture of the networked area, regarding the attribute(s) being monitored. Typically, data generated by individual nodes are sent to a powerful node, known as Base Station (BS) or sink. Here the data can be aggregated and forwarded to the user, possibly using the Internet, where the data can be further analysed and useful information can be extracted. They can be deployed over a battle field for military surveillance, emergent environments for search and rescue environment monitoring health care applications etc. A sensor node is typically powered by a battery, and energy saving is of critical importance since these networks are usually deployed in unfriendly remote environments. This isolation makes changing or charging the battery expensive, if not impossible. Thus, maximizing the lifetime of a sensor network, keeping an acceptable performance level, has seen substantial interest. There are number of ways in which the lifetime can be improved. This paper addresses this issue by the use of relay nodes in cluster based sensor network. The next section deals with the survey of the related work carried out in this field. In section 3, a description of the protocol has been given and section 4 deals with the results and discussions. The paper concludes with section 5 .

\section{RELATED WORK}

Low-Energy Adaptive Clustering Hierarchy (LEACH)[1] is a clustering-based protocol that minimizes energy dissipation in sensor networks. The purpose of LEACH is to randomly select sensor nodes as cluster heads, so the high energy dissipation in communicating with the base station is spread to all sensor nodes in the sensor network. This protocol offers no guarantee about the placement and/or number of cluster head nodes. Since the clusters are adaptive, obtaining a poor clustering set-up during a given round will greatly affect overall performance.

In BCDCP [2], the main activities are cluster setup, cluster head selection, $\mathrm{CH}$-to- $\mathrm{CH}$ routing path formation, and schedule creation for each cluster. During each setup phase, the base station receives information on the current energy status from all the nodes in the network. Based on this feedback, the base station first computes the average energy level of all the nodes, and then chooses a set of nodes, denoted $\$$, whose energy levels are above the average value. Cluster heads for the current round will be chosen from the set $\$$, which ensures that only nodes with sufficient energy get selected as cluster heads, while those with low energy can prolong their lifetime by performing tasks that require low energy.

The deployment of relay nodes in hierarchical architecture was first proposed in 2003.In [3], Gupta and Younis focused on the issue of load-balancing and proposed an algorithm for load-balanced-clustering of hierarchical sensor networks. They called the relay nodes as gateway nodes. Through experimental results, the authors claimed that, as compared to the shortest-distance approach, their method have shown better performance for both in terms of standard deviation of load per cluster and average communication energy per cluster. In the paper by Pan et al.[ 4 ] the relay nodes are called as aggregation nodes (AN), and an attempt to maximize the topological network lifetime of sensor networks was made. They have proposed a two-tier sensor network model where sensor nodes lie in the lower tier and the Application Nodes (ANs) \& and the Base Stations (BSs) lie in the upper tier. Their algorithms are based on Computational Geometry that finds the optimal locations of the BSs under three topological definitions, $\mathrm{N}$-of-N lifetime (i.e. mission fails if any gateway node dies), K-of-N lifetime (i.e. mission survives if a minimum of $\mathrm{K}$ gateway nodes are alive) and $\mathrm{m}$-in-K-of-N lifetime (i.e. mission survives if all $\mathrm{m}$ supporting nodes and overall a minimum of $\mathrm{K}$ gateway nodes are alive), all based on the criticality of the mission. They have established theoretical upper and lower bounds on the maximal topological lifetime of sensor network. Tang and Xue in [5], focused on optimizing the number of relay nodes that can be used in a sensor network such that the connectivity and faulttolerance can be ensured. In a two-tier network. Here sensor nodes lie in the lower tier and relay nodes lie in the upper tier. These relay nodes are used as cluster heads and are responsible for collecting data from the sensor nodes of their respective cluster, aggregating received data, forming connected topology and transmitting the data towards the sink using multi-hop routing. The authors have formulated a faulttolerant scheme for such networks for finding the minimum number of relay nodes such that each sensor node is 
connected with at least two relay nodes and the relay nodes network itself is two connected. They also have proposed a polynomial-time approximation algorithm to solve the problem and claimed that the worst-case performance of their solution is bounded by $\mathrm{O}(\mathrm{D} \log \mathrm{n})$ times of the size of an optimal solution. In [6], Patel et al. addressed the placement problem of sensor nodes, relay nodes and base station in sensor networks and formulated a solution to achieve minimal number of sensor nodes, minimal total cost and energy consumption as well as maximal energy utilization and lifetime of the network. In [7], Hou et al. (2005) focused on prolonging the lifetime of sensor networks with energy provisioning and deploying relay nodes within the networks.

\section{EERMC PROTOCOL}

\subsection{Design}

Consider a two dimensional network area where large numbers of energy constrained sensor nodes are randomly deployed. The sink node or base station, BS is placed far away from all the sensor nodes. The following assumptions are made:

- Sensor nodes can estimate their positions with the help of few properly placed nodes which are embedded with the GPS devices

- All nodes are homogeneous in terms of computation, communication capabilities and power resources.

- Each sensor node has power control capabilities to vary their transmission power depending on distance to receiver.

The BS broadcasts the control messages to the sensors which helps it to synchronize the local time or clock with the BS. To determine the position of the sensor node with respect to the base station, the BS scans the entire region. The base station sends control messages containing the current directionality information and the transmit power level (indicating the relative distance between the sensor and the base station) through successive scans of the network. A nodes location is thus determined by the directionality of the last BS transmission (called sector number $\mathrm{SN}$ ), as well as the lowest power level that it can receive from the base station (called $\mathrm{TN})$. We therefore define the id of a sensor to be the 2-tuple $\{\mathrm{SN} ; \mathrm{TN}\}$. The index associated with the direction of the current transmission is $\mathrm{SN}$ and $\mathrm{TN}$ is the index associated with the current transmit power. The field ScanNum is the number of the current scan. When a sensor receives multiple control messages with the same ScanNum, its id is chosen as below:

\section{\{ $\mathrm{SN}=\max \left\{\mathrm{SN}_{\mathrm{i}} \cdot \mathrm{rcvd}\right.$ ctrl msgs with ScanNum $\}$}

$\mathrm{TN}=\min \left\{\mathrm{TN}_{\mathrm{i}} \cdot \mathrm{rcvd}\right.$ ctrl msgs with ScanNum $\}$

$$
\text { \} }
$$

Initially, the directional antenna broadcasts a message with $\{\mathrm{SN}=1, \mathrm{TN}=1\}$ to all nodes with in this region. Sensors located in this region will then obtain there id a $\{\mathrm{SN}, \mathrm{TN}\}=$ $\{1,1\}$. Subsequently, the base station adjusts its transmit power to the next higher level and broadcasts a message with location information $\{\mathrm{SN}=1, \mathrm{TN}=2\}$. Those sensor nodes that have acquired id $\{1,1\}$ from the earlier transmission will not change their id. By repeating this procedure, all sensor nodes can derive their ids via receiving broadcast control messages from the base station. During this process, as there is no need for any sensor node to exchange any information with its neighbors, power is saved and the complexity has been effectively shifted from sensor node to the BS. Based on received information, BS selects the relays and primary set of Cluster Head $(\mathrm{CH})$ nodes using cluster head selection algorithm described later and broadcasts the selected CHs' ID to all the nodes in network. The remaining operation of EERMC protocol is divided into two different phases: the setup phase and the data transmission phase .In the Setup phase a node that finds its ID in the $\mathrm{CH}$ list becomes a $\mathrm{CH}$ for the current round and broadcasts the advertise packet using CSMA MAC protocol. All non-CH nodes receives this packet from neighboring $\mathrm{CH}$ nodes and decides to which cluster it belongs based on Received Signal Strength Indicator (RSSI) and transmits joining request to chosen $\mathrm{CH}$. After a predefined time, based on the received JOIN-PK messages each $\mathrm{CH}$ node creates the TDMA schedule for its cluster members and broadcast the schedule along with specified spreading code to overcome the intra cluster interference in network.

In the Data Transmission Phase, data transmission in intracluster and $\mathrm{CH}$ to BS takes place using TDMA and CSMA MAC protocols, respectively. Each cluster member node transmits its monitored data to local base station $(\mathrm{CH})$ during its allocated time slot. Once $\mathrm{CH}$ nodes receive the sensed data from their cluster members, each $\mathrm{CH}$ node performs the data aggregation on the correlated data and transmits the compressed data to BS.

\subsection{Relay Nodes Selection Based On Energy and Density}

Let number of $\mathrm{CH}$ in the network $=\mathrm{k}$

For each $\mathrm{CH}$ calculate the average percentage of energy spent (AES) and the percentage of area covered during transmission (PAC). The area considered is twice the radius of the node. The ratio of these percentages is used to decide if the relay nodes are required or not.

Decision $\mathrm{D}=\mathrm{AES} / \mathrm{PAC}$.

$$
\begin{gathered}
\text { IF D } \geq 1 \quad \text { Relay necessary } \\
\text { ELSE. } \quad \text { Relay not required }
\end{gathered}
$$

\subsection{Determination of Relay Node Position}

In the network source node is represented as $\mathrm{A}(\mathrm{x}, \mathrm{y})$ and destination as $\mathrm{B}(\mathrm{x}, \mathrm{y})$. The co-ordinates of source, destination and relay are represented as $A_{x}, A_{y}, B_{x}, B_{y}, R_{x}$ and $R_{y}$ respectively. Let the transmission radius of relay be $T_{R}$.

Represent the line joining the source to destination as $\mathrm{y}=\mathrm{mx}+\mathrm{c}$.

Algorithm

Input: Source, destination, no. of relay

Output: position of relay nodes

1. for $\mathrm{i}$ from 1 to $\mathrm{k}$

2. if $A_{x}<B_{x}$

for $x$ from $\left(A_{x}+T_{R} * \cos \left(\tan ^{-1}(m)\right)\right)$ to $B_{x}$ for every $T_{R} * \cos$ $\left(\tan ^{-1}(\mathrm{~m})\right)$

$R_{x}(i)=x$,
$R_{y}(i)=m *\left(T_{R}\right)_{x}+c$

3. else 
for $x$ from $\left(A_{x}-T_{R} * \cos \left(\tan ^{-1}(m)\right)\right)$ to $B_{x}$ for every $T_{R} * \cos$ $\left(\tan ^{-1}(\mathrm{~m})\right)$

$\mathrm{R}_{\mathrm{x}}(\mathrm{i})=\mathrm{x}$,

$\mathrm{R}_{\mathrm{y}}(\mathrm{i})=\mathrm{m} *\left(\mathrm{TR}_{\mathrm{R}}\right)_{\mathrm{x}}+\mathrm{c}$

\section{SIMULATIONS AND RESULTS}

To evaluate the performance of EERMC protocol, we have simulated a network generated over $100 \mathrm{mx} 100 \mathrm{~m}$ area in MATLAB. We randomly deployed $\mathrm{N}=100$ sensor nodes in network area with initial energy of 50 joules in each node. The BS was placed at $(50,175)$ and is assumed to be powered by external source. The necessary communication parameters are listed in Table.1 [8]

Table 1: Communication Parameters

\begin{tabular}{|l|l|l|l|}
\hline $\mathrm{X}_{\mathrm{m}}=100$ & $\mathrm{Fc}=900 \mathrm{MHz}$ & $\mathrm{M}_{1}=40 \mathrm{~dB}$ & $\mathrm{P}_{\mathrm{IFA}}=3 \mathrm{~mW}$ \\
\hline $\mathrm{Y}_{\mathrm{m}}=100$ & $\mathrm{~B}=10 \mathrm{kHz}$ & $\alpha=0.45$ & $\mathrm{P}_{\mathrm{mix}}=30.3 \mathrm{~mW}$ \\
\hline $\mathrm{N}=100$ & $\eta=3.6$ & $\mathrm{~b}=2$ & $\mathrm{P}_{\mathrm{Filt}}=2.5 \mathrm{~mW}$ \\
\hline $\mathrm{BSx}=50$ & $\mathrm{G}_{\mathrm{t}} \mathrm{G}_{\mathrm{r}}=0 \mathrm{dBi}$ & $\mathrm{P}_{\mathrm{b}}=10^{-3}$ & $\mathrm{P}_{\mathrm{LNA}}=20 \mathrm{~mW}$ \\
\hline BSy=175 & $\begin{array}{l}\mathrm{N}_{0}=-174 \\
\mathrm{dBm} / \mathrm{Hz}\end{array}$ & $\mathrm{P}_{\mathrm{ADC}}=20 \mathrm{~mW}$ & $\mathrm{~N}_{\mathrm{f}}=10 \mathrm{~dB}$ \\
\hline
\end{tabular}

Figure 1 and 2 gives the cooperative relay position in network such that it balances the energy dissipation in cluster which are near and far from BS, minimize the total energy consumption in the network respectively. Certainly, optimum relay positions minimize the energy imbalanced hotspots in the network through approximately balancing the energy consumption

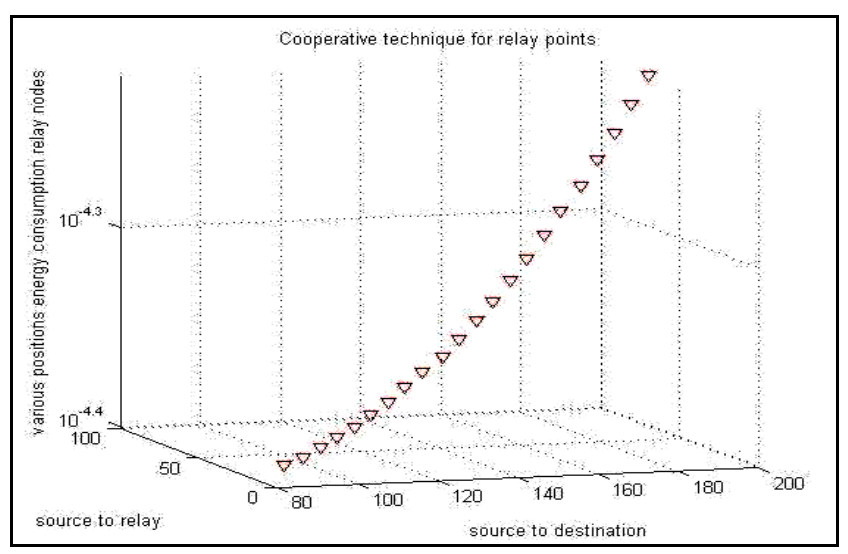

Figure 1: Energy consumption for various relay positions

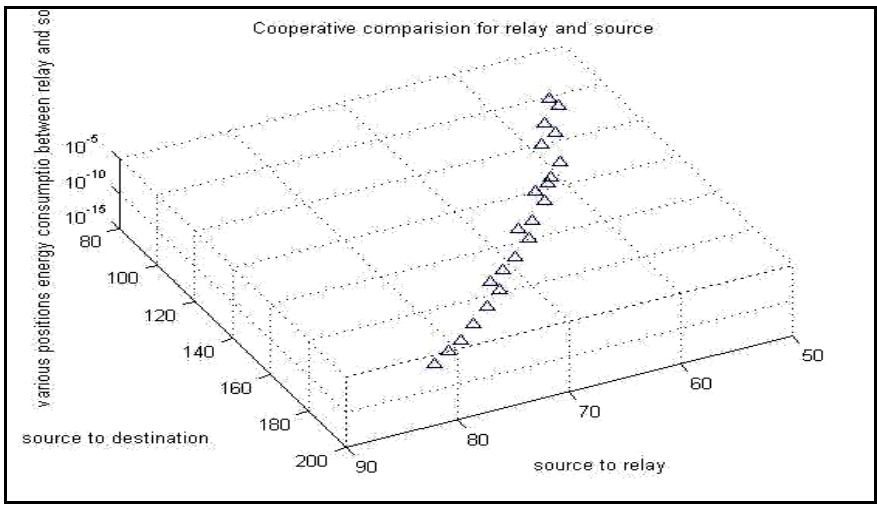

Figure 2: Optimum relay positions

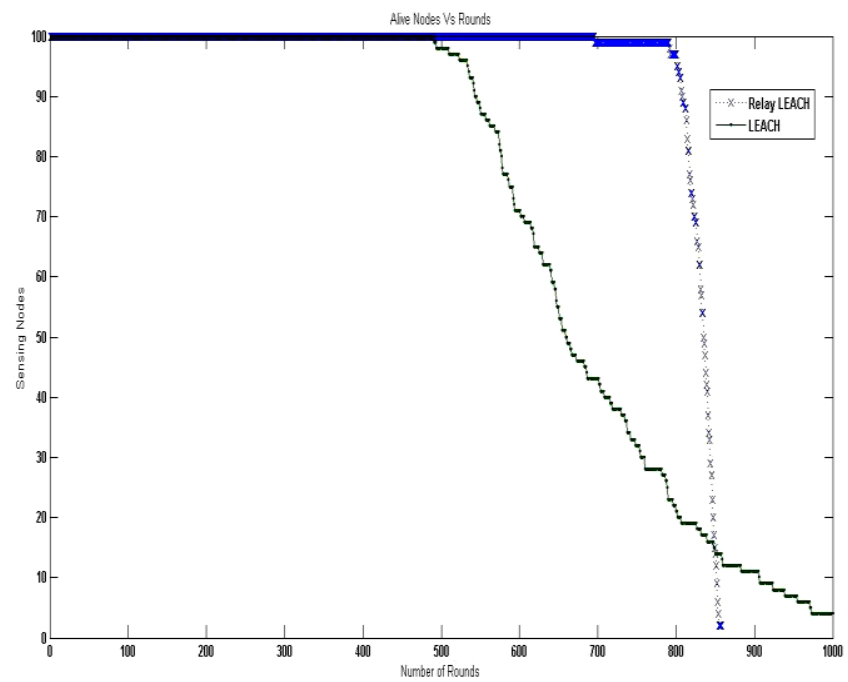

Figure 3: Network Lifetime Graph

Figure 3, shows the Network Lifetime Graph. It is observed that the proposed EERMC protocol has the death of its first node after 700 rounds compared to that of LEACH where node dies at about 500 rounds. Further in LEACH the nodes far away from the base station die faster than the closer ones. The lifetime, defined as the time of death of last node, is less than the LEACH but it shows that the data collection is throughout the network.

Figure 4 shows the variation of mean residual energy of the node with respect to the number of data collection rounds for LEACH and EERMC protocols. The graph shows that the remaining energy is higher and hence the lifetime is increased when compared with the LEACH protocol. 


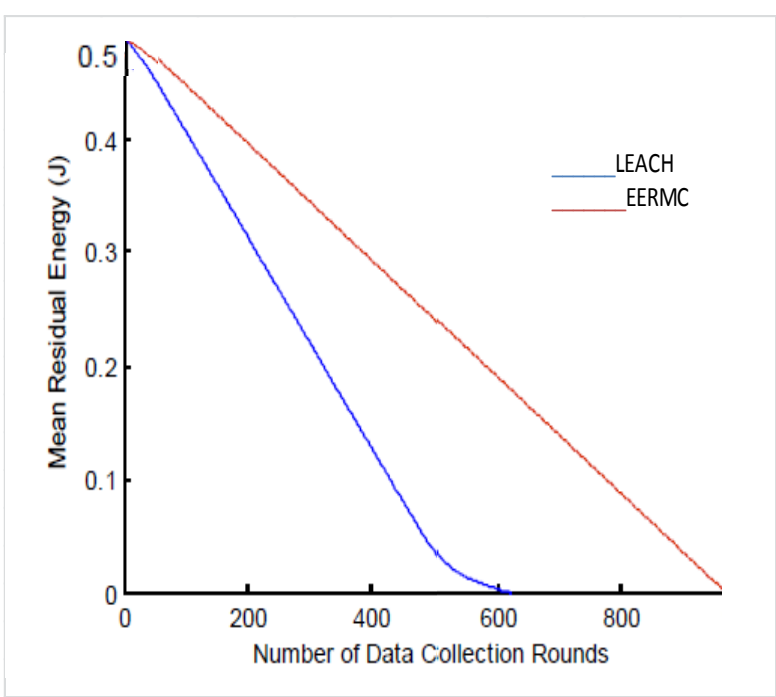

Figure 4: Variation of Mean Residual Energy

Figure 5 shows a graph that illustrates the variation in the energy consumed per round with respect to cluster size and network diameter. The $\mathrm{x}$-axis, $\mathrm{y}$-axis, and $\mathrm{z}$-axis represent the network diameter, the cluster size, and the energy consumed in one round, respectively.. The number of data frames in one iteration is 10,000 and the number of clusters $=5$. As expected, the graph shows that energy consumption is reduced when the cluster size is increased since re-clustering is not needed frequently and the energy is conserved.

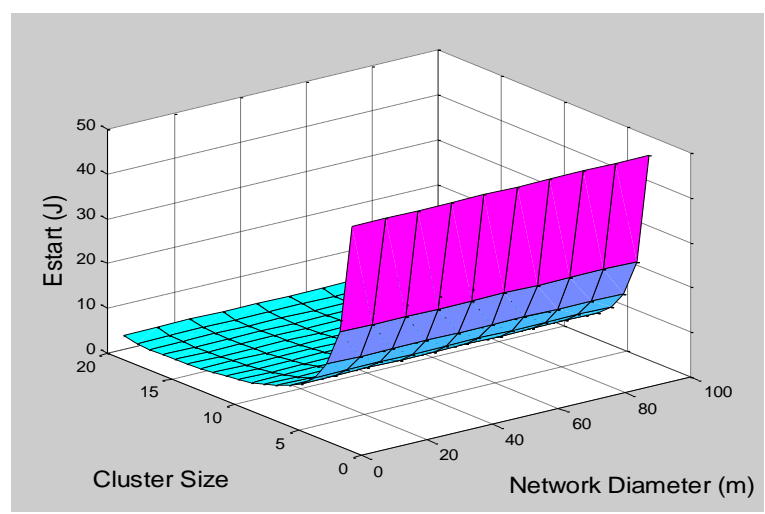

Figure 5: Graph that illustrates the variation in the energy consumed per round with respect to cluster size and network diameter

\section{CONCLUSION}

The main objectives of this work were to design an energy efficient protocol that balance the energy consumption in clusters which are placed at various distances from the sink and to select the relay nodes such that dual-hop MISO technique with relay nodes could be implemented to increase the lifetime. Comparing the proposed protocol life time with the standard LEACH protocol and BCDCP, we can see a large improvement. This is because of the linear decrease in the energy of the network.

\section{ACKNOWLEDGMENTS}

The authors would like to thanks to BMS College of Engineering, PES Institute of Technology and VGST(Vison Group of Science and Technology) Gov. of Karnataka, India for providing infrastructure facilities .

\section{REFERENCES}

[1] W. Heinzelman, A. Chandrakasan, and H. Balakrishnan, "An application-specific protocol architecture for wireless microsensor networks," Wireless Communications, IEEE Transactions on, vol. 1, no. 4, pp. $660-670$, Oct 2002 .

[2] S. Muruganathan, D. Ma, R. Bhasin, and A. Fapojuwo, "A centralized energy efficient routing protocol for wireless sensor networks," Communications Magazine, IEEE, vol. 43, no.3, pp.8 - 13, March 2005

[3] G. Gupta and M. Younis, " Load-balanced clustering of wireless sensor networks", IEEE International Conference on Communications, volume 3, pages 18481852,2003

[4] J. Pan, Y. T. Hou, L. Cai, Y. Shi, and S. X. Shen, "Topology Control for Wireless Sensor Networks", Proceedings of Ninth Annual International Conference on Mobile Computing and Networking, pages 286-299, 2003.

[5] B. Hao, J. Tang, and G. Xue, "Fault-tolerant relay node placement in wireless sensor networks: formulation and approximation", Workshop on High Performance Switching and Routing (HPSR), pages 246-250, 2004.

[6] M. Patel, R. Chandrasekaran, and S. Venkatesan, "Energy Efficient Sensor, Relay and Base Station Placements for Coverage, Connectivity and Routing", 24th IEEE International Performance Computing and Communications Conference (IPCCC), pages 581 - 586, 2005.

[7] Y. T. Hou, Y. Shi, H. D. Sherali, and S. F. Midkiff, "On Energy Provisioning and Relay Node placement for Wireless Sensor Networks", IEEE Transactions on Wireless Communications, 4(5):2579-2590, 2005.

[8] S. Cui, A. Goldsmith, and A. Bahai, "Energy-efficiency of mimo and cooperative mimo techniques in sensor networks," IEEE Journal on Selected Areas in Communications, vol. 22,no. 6, pp. 1089 - 1098, Aug 2004. 